\title{
A NOVEL COMPACT THRESHOLD ČERENKOV DETECTOR
}

\author{
W.J.C. Okx; C.W.E. van Eijk and R. de Vries \\ Delft University of Technology, Delft, The Netherlands
}

\begin{abstract}
In a number of particle physics experiments fast discrimination between pions and kaons is required. At high momenta a Cerenkov detector is the most appropriate for that purpose. In general a limited amount of space is available for such a detector, which imposes severe limitations on the design. In this paper we present a detector in which the Cerenkov UV-light is produced in a thin layer $(4 \mathrm{~cm})$ of a liquid-FC72 radiator in which a row of $\varnothing 2 \mathrm{~cm}$ quartz tubes is immersed. The light is detected in these tubes. Inside a tube a wire is stretched along the axis as anode and parallel wires on the inner circumference function as the cathode. The tubes are operated with TMAE vapour without additional counting gas, so that each tube acts as an elementary low pressure proportional chamber. Using this principle the responce of charged particles passing the detector is suppressed.

Computer simulations have shown that good pion/kaon discrimination can be obtained. After a pilot study with one 'tube light detector' we constructed a small $20 \times 20 \mathrm{~cm}^{2}$ prototype Cerenkov detector which will be described.
\end{abstract}

\section{Introduction}

In a number of particle physics experiments discrimination between charged pions and kaons is required. In general the amount of space in an experimental setup for a detector which can cope with this problem is limited. In this paper we will discuss the case of the experiment on $C P$ breaking by the CP LEAR collaboration [1]. The experimental setup consists of a solenoid with many detectors inside.

For the $\pi / K$ discriminating device, an annular region with a radius of $\approx 0.60 \mathrm{~m}$, a thickness of $6 \mathrm{~cm}$ and a length of $2.80 \mathrm{~m}$ is available. Within these dimensions and with pion momenta up to $900 \mathrm{MeV} / \mathrm{c}$, it is not possible to identify the particles by means of a time of flight method. A ring imaging Cerenkov detector [2] would positively identify the charged kaons but it is difficult to construct such a device within the given dimensions. Consequently, a threshold Cerenkov detector is the best choice. Such a

\footnotetext{
+lidlin of the knwal Netherlands Academy of Arts and Sciences
}

detector will identify the faster particles, i.e. the charged pions, thus marking the not detected particles as kaons. In a conventional threshold Čerenkov detector, the light produced in the radiator is converted to the red wavelength region with a wavelength shifter and subsequently read out through long light guides by means of photomultipliers (PM). These PMs have to be located outside the magnetic field, which complicates the detector design. Cerenkov light can also be detected by means of a Multi Wire Proportional Chamber (MWPC) filled with TMAE vapour. In this paper we describe a novel design of a threshold Cerenkov detector based on this method.

\section{Detector Principle}

A Čerenkov detector produces a signal when the velocity $\beta$ of the particles traversing the detector is above a threshold value $\beta_{t}$ given by the relation $\beta_{\ell}=n^{-1}$, in which $n$ is the refractive index of the radiator. For a good $\pi / K$ discrimination in the CP LEAR experiment the threshold value has to be $\beta_{t}=0.82$, i.e. $n=1.22$. For kaons this value of $\beta_{\ell}$ corresponds to $710 \mathrm{MeV} / \mathrm{c}$ which is close to the endpoint of the charged kaon spectrum. For pions this value corresponds to $200 \mathrm{MeV} / \mathrm{c}$, i.e. a value low compared to the maximum pion momentum of $900 \mathrm{MeV} / \mathrm{c}$. Thus, as opposed to kaons, almost all pions produce a signal in the detector.

Of the non cryogenic solids and liquids, FC72 $\left(C_{t i} F_{1.1}\right)$ has an index of refraction of $n=1.27$ which draws nearest to the value of 1.22 . Therefore $\mathrm{FC} 72$ is the best radiator for this detector. Liquid $\mathrm{FC} 72$ has good transmission characteristics in the UV part of the spectrum so that it is possible to detect the light from this radiator in an MWPC with TMAE vapour. Fig. 1 shows the transmission of FC72 and quartz and the quantum efficiency of TMAE [3]. To retain the UV transmission, the $\mathrm{FC} 72$ has to be circulated and filtered continuously to remove oxygen and water vapour [4]. From the overlap of the curves, it is clear that an FC72 radiator separated by a quartz window from a TMAE-filled wire chamber is an interesting combination.

In imaging Cerenkov detectors the disturbing signal from the passing charged particle is removed during the analysis using the imaging capabilities of these detectors (e.g. see 
Transmission

Quantun Ifficiency

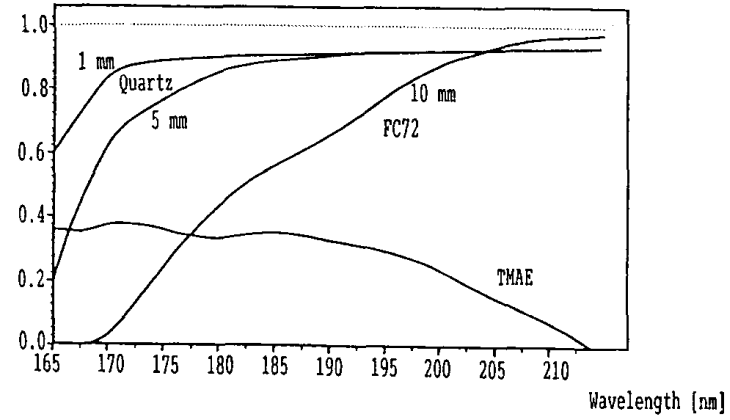

Fig.1. Transmission of $1 \mathrm{~mm}$ and $5 \mathrm{~mm}$ of quartz, and of $10 \mathrm{~mm}$ of FC72 and the quantum efficiency of TMAE as a function of wavelength.

[2]). In the present study the particle signal is suppressed by operating the proportional chamber at the vapour pressure of TMAE. In a trajectory of $2 \mathrm{~cm}$ of counting gas at a pressure of $2.6 \mathrm{mbar}\left(\mathrm{T}=45^{\circ} \mathrm{C}\right)$, minimum ionizing particles will release at most a few electrons. By putting the detection threshold at about 6 primary electrons as indicated in fig. 3 , the efficiency of the low pressure proportional chamber for charged particles becomes very small.

Another advantage is the fast response of a low pressure wire chamber. The signal can be included in the trigger or used as a veto signal.

We have investigated two possible detector geometries. In fig. $2 \mathrm{a}$ the radiator and the wire chamber are separated by a quartz plate. Because of the pressure difference between the two parts of $\approx 1$ bar, the quartz plate has to be $5 \mathrm{~mm}$ thick at a width of only $10 \mathrm{~cm}$. However, quartz also acts as a radiator and, as a consequence of its refractive index of $n \approx 1.58$, it has a threshold at $\beta=0.63$.
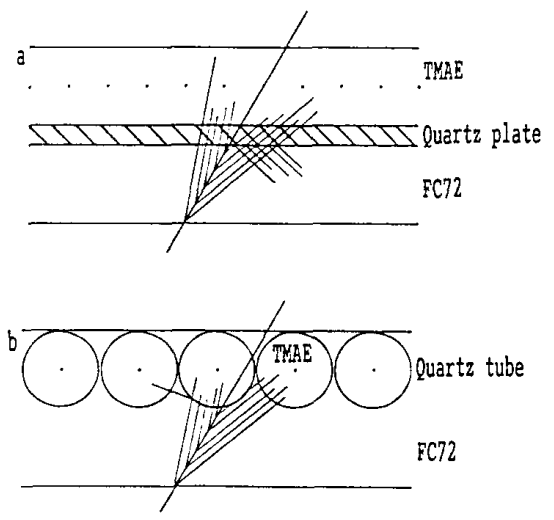

Fig.2. Two possible detector systems: a. 'Plate detector': a $5 \mathrm{~mm}$ quartz plate as entrance window for a light sensitive wire chamber, b. 'Tube detector': a system of long quartz tubes; each tube functions as a proportional counter.
Thus it will also produce light for most of the charged kaons. To reduce the amount of quartz in the system, we also investigated the detector geometry shown in fig. $2 \mathrm{~b}$. The wire chamber is divided into single cells, and every cell is located inside a quartz tube which is immersed in FC72. The mechanical properties of a tube are much better than those of a flat plate, so that the 'window' thickness can be reduced. Quartz tubes with an outer diameter of $20 \mathrm{~mm}$ and a wall thickness of $1 \mathrm{~mm}$ are commercially available in lengths of $1.50 \mathrm{~m}$ (Suprasil). These tubes can easily withstand the $\approx 1$ bar overpressure of FC72.

\section{Simulation}

For the two detector geometries mentioned earlier, we performed Monte Carlo simulations of the production of photoelectrons in the TMAE by Cerenkov light from particles in the $\beta$ range $0.6-1.0$. From these simulations, which are discussed in an earlier paper [5], we recall fig. 3 to summarize the results.

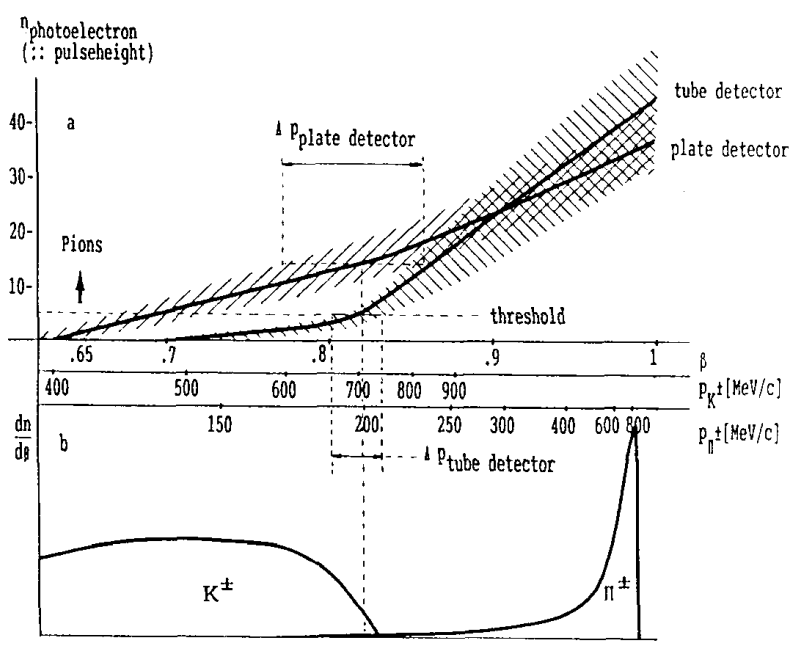

Fig.3. Pion and kaon distribution in the CP LEAR experiment (b) and the simulated pulse height as a function of $\beta$ of the particles (a). The shaded areas in (a) account for the angular distribution of the particles and the detection statistics of the produced UV-light.

The maximum number of photoelectrons at $\beta=1$ is 45 . The photoelectrons in the range $\beta=0.63-0.79$ are purely due to Cerenkov effect in quartz; FC72 starts to contribute at $\beta=0.79$. In the tube detector the contribution from quartz to the signal is significantly lower than in the plate detector. In addition, starting from $\beta=0.79$, the number of photoelectrons increases much faster with $\beta$ in the tube detector as a consequence of the better detection efficiency of the tubes for Cerenkov light. The low photoelectron yield at $\beta \approx 0.82$ and the steep rise at higher $\beta$ values offer the possibility of a relatively sharp discrimination as indicated in the figure. 


\section{Detector design}

A threshold Cerenkov detector which will fit within the dimensions mentioned in the Introduction can be composed of e.g. 16 modules with a width of $\approx 0.24 \mathrm{~m}$ (12 tubes) and a length of $2.80 \mathrm{~m}$. The signal and the 'plumbing' for FC72 and TMAE can easily be led through the end caps of the magnet. Attention has to be given to an appropriate heat insulation.

To test the performance of this design, we have constructed a small module with 10 quartz tubes (fig.4). The quartz tubes $\left(\emptyset 20 \times 1 \mathrm{~mm}^{2}\right.$, length $\left.240 \mathrm{~mm}\right)$ are sealed at one side.

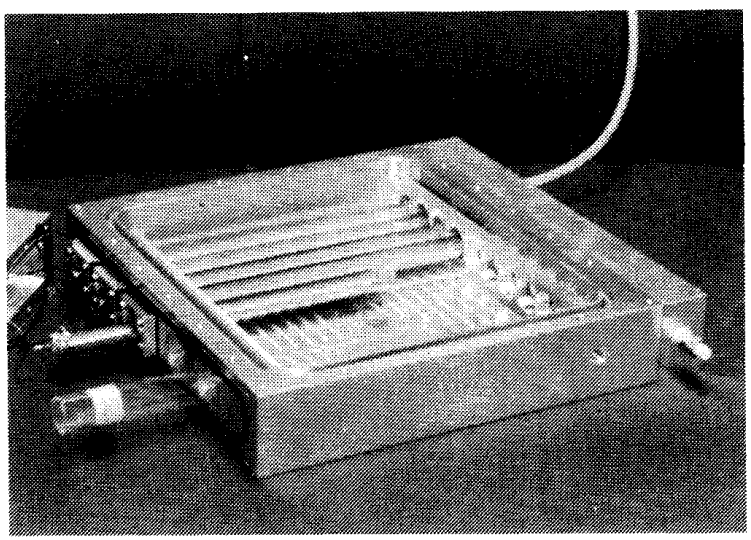

Fig.4. The Cerenkov detector module. Five quartz tubes $(\varnothing 20 \times 1 \mathrm{~mm} 2$, length $240 \mathrm{~mm})$ are clamped with O-rings into holes in the detector body. An anode-cathode cell is slid partly into a tube. An aluminium cover is partly visible on the left. The cover on the right is in position.

They are slid into the aluminium detector body, alternately through holes on two opposite sides. By means of viton O-rings, they are clamped vacuum tight to the detector body, thus separating the TMAE and FC72 compartments. From the two opposite sides of the module the anode-cathode cells can now be slid into the tubes. The basic element of a cell is a half cylinder $(\varnothing 17 \mathrm{~mm})$ of $0.5 \mathrm{~mm}$ stainless steel. This cylinder functions as mechanical support, as a part of the cathode and as a shield for light produced in the 'rear' side of the quartz tube. Along the axis of the cylinder, a $\varnothing 20 \mu \mathrm{m}$ gold plated tungsten anode wire is stretched and along the open half of the cylinder $\varnothing 50 \mu \mathrm{m}$ stainless steel cathode wires are located. The cathode wires also prevent charge build up on the quartz wall. After assembling all tubes, an aluminium cover is mounted on each side of the module to close the open quartz tubes. After evacuation the quartz tubes are filled with TMAE vapour. In order to have an optimum detection efficiency for the UV light, the temperature of the system will be raised to $45^{\circ} \mathrm{C}$. At this temperature the $1 / \mathrm{e}$ absorption length of UV light in TMAE is $\approx 6 \mathrm{~mm}$. Thus a good detection efficiency in the $20 \mathrm{~mm}$ diameter tubes is guaranteed. The elevated temperature can easily be maintained by heating the circulating FC72.
The entrance window of the detector $(2 \mathrm{~mm} \mathrm{Al})$ is sealed with an O-ring, so that the filtered and heated FC72 can be circulated in a closed air tight system through the detector body over the quartz tubes.

\section{Experimental Results}

The detector has been tested by observing UV scintillation light from a small $\mathrm{BaF}_{2}$ crystal $(\varnothing 13 \mathrm{~mm}$, thickness $5 \mathrm{~mm}$ ) optically coupled to a quartz tube and irradiated with a ${ }^{90} \mathrm{Sr}$ source. $\mathrm{BaF}_{2}$ is an easy and reliable light source in the required wavelength region. Earlier experiments have shown that an irradiated $\mathrm{BaF}_{2}$ crystal, located inside a wire chamber, produces at most 20 primary electrons per $\mathrm{MeV}[6]$.

From the poor coupling between the flat crystal and the curved surface of the quartz tube one expects an electron yield of about one. Thus an adequate test is obtained of the gain of the structure and possible photon feedback effects. During the test all quartz tubes were mounted but only three of them had been provided with an anodecathode cell. The detector has been operated with TMAE vapour at room temperature $\left(23^{\prime} \mathrm{C}\right)$ so that the $1 / \mathrm{e}$ absorption length is $25 \mathrm{~mm}$ (to be compared to $6 \mathrm{~mm}$, when the detector is heated to $45^{\circ} \mathrm{C}$ by the $\mathrm{FC} 72$ ). The gas amplification region around the anode wire is rather expanded at the lower operating temperature. Consequently there is a large spread in the single electron signal response as the photons are absorbed throughout the tube. Fig.5 shows the scintillation light spectrum (a) and the background spectrum (b). The background spectrum has been recorded with the $\mathrm{BaF}_{2}$ crystal and "'Sr source in position but with a $5 \mu \mathrm{m}$ Mylar foil between crystal and quartz tube. The foil prevents UV light from entering the quartz tube but does not influence the radiation condition from the ${ }^{90} \mathrm{Sr}$ source incident to the tube. Above the lower level threshold at about channel 30 , both spectra have nearly the same shape. Therefore it is plausible that we measure

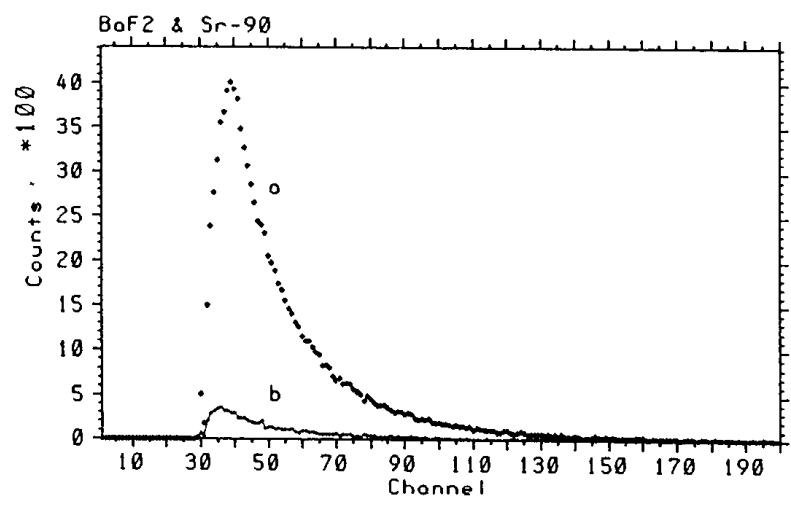

Fig.5. Spectra recorded with the quartz tubes: a) UV-light and background and b) background. 
single photoelectron spectra as expected from the poor light coupling between the crystal and the quartz tube. The tests have been repeated at 9 different positions of $\mathrm{BaF}_{2}$ crystal on the quartz tubes, all with a similar result. Thus we have demonstrated that the gas amplification is adequate and that photon feedback effects, which we observed at higher values of the amplification, do not pose a problem. Actually the amplification may even be reduced in tests with a particle beam. At present we are assembling the whole test system. After filling the detector body with FC72, the module will be tested with cosmic rays at room temperature and at $45^{\circ 1} \mathrm{C}$ and, later on, in a pion beam with variable momentum.

\section{References}

1 CP LEAR Collaboration, The LEAR $\mathrm{K}^{0}$ Experiment, Il Nuovo Cimento 102A, N1, 127 (1989).

2 R. Arnold, P. Baillon, J.D. Berst, H.J. Besch, M. Bosteels, E. Cristophel, Y. Giomataris, J.L. Guyonnet, G. Passardi, J. Seguinot, J. Tocqueville, D. Toet and T. Ypsilantis, Nucl. Instr. and Meth. A252, 188 (1986).

3 R.A. Holroyd, J.A. Preses and C.L. Woody, Proceedings of 23rd Int. Conf. on High Energy Physics, Berkeley, Cal., USA, July 1986.

4 M. Bosteels and G. Lenzen, Technical Note DELPHI 86-77, RICH-20, CERN, Geneva, Switzerland, 1986.

5 W.J.C. Okx, C.W.E. van Eijk, M. Looman and N.W. Tanner, Nucl. Instr. and Meth. A273, 548 (1988).

6 C.L. Woody, Technical note No. 120, Physics Department Brookhaven National Laboratory (Associated Universities Inc. Upton, New York 11973,1986). 